\title{
Polarized proton parameters for the 2015 PP-on-Aluminum setup in RHIC
}

\author{
C. J. Gardner
}

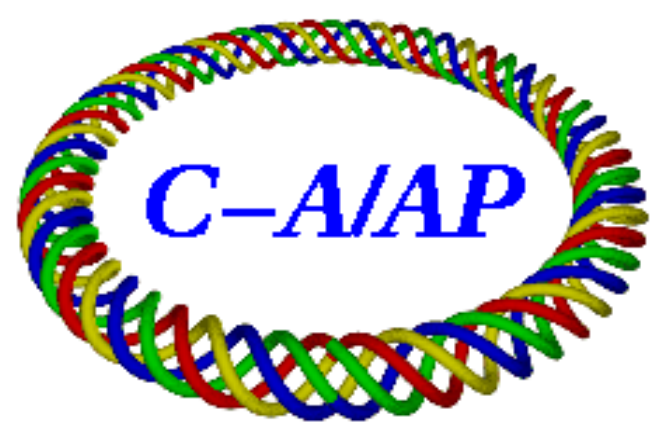

\section{Collider-Accelerator Department Brookhaven National Laboratory Upton, NY 11973}

\section{U.S. Department of Energy \\ Office of Science, Office of Nuclear Physics}

Notice: This document has been authorized by employees of Brookhaven Science Associates, LLC under Contract No. DE-SC0012704 with the U.S. Department of Energy. The United States Government retains a nonexclusive, paid-up, irrevocable, world-wide license to publish or reproduce the published form of this document, or allow others to do so, for United States Government purposes. 


\title{
Polarized Proton Parameters for the PP-on-Aluminum Setup in RHIC
}

\author{
C.J. Gardner
}

October 2, 2015

Proton mass-energy equivalent $m_{p} c^{2}=938.272046(21) \mathrm{MeV}[1]$

Proton $\mathrm{g}$ factor $g_{p}=5.585694713(46)[1]$

Proton $G=\left(g_{p}-2\right) / 2=1.79284735650$

Booster Injection Kinetic Energy $=200 \mathrm{MeV}$

Booster Extraction $G \gamma=4.5$

Nominal Booster radius-of-curvature $\rho=13.8656 \mathrm{~m}$

Nominal AGS radius-of-curvature $\rho=85.378351 \mathrm{~m}$

Ags Injection $G \gamma=4.5$

Ags Extraction $G \gamma=45.5$

Nominal RHIC Circumference: $C_{r}=3833.845181 \mathrm{~m}$

RHIC Store $G \gamma=198.5$ 
$\underline{\text { Circumference Shifts }}$

The RHIC circumference shift due to snakes $[2,3]$ is

$$
\delta L=26.1928376473988 /(B \rho)^{2}
$$

where the units of $\delta L$ and $B \rho$ are $\mathrm{m}$ and Tm respectively.

Shift at $G \gamma=45.5$ due to snakes: $\delta L=4.1582 \mathrm{~mm}$

Shift at $E=100 \mathrm{GeV}$ due to snakes: $\delta L=0.2354 \mathrm{~mm}$

Shift at $G \gamma=191.5$ due to snakes: $\delta L=0.2344 \mathrm{~mm}$

Shift at $G \gamma=198.5$ due to snakes: $\delta L=0.218156 \mathrm{~mm}$

Shift at $E=250 \mathrm{GeV}$ due to snakes: $\delta L=0.0377 \mathrm{~mm}$

There are also circumference shifts $\delta D$ due to unequal ion rigidities in the DX magnets.

The total RHIC circumference shift is $\underline{\delta C=\delta L+\delta D}$.

Ags Circumference at Extraction $=4\left(C_{r}+\delta C\right) / 19$.

The DX magnets give shift $\delta D=10.367846 \mathrm{~mm}$ at blue injection [4].

The DX magnets give shift $\delta D=8.995322 \mathrm{~mm}$ at blue store [4].

\section{References}

[1] P.J. Mohr and B.N. Taylor, "Values of Fundamental Physical Constants", Physical Constants, Physical Reference Data, www.nist.gov.

[2] W. MacKay, "Path Length through Helical Snakes and Rotators", C-A/AP/Note 140, March 2004.

[3] Formula (1) follows from those derived in [2]. It is used by Al Marusic to obtain path lengths in the RHIC snakes.

[4] As calculated by Al Maursic. 
Table 1: Polarized Protons in Booster

\begin{tabular}{|c|c|c|c|}
\hline Parameter & Injection & Extraction & Unit \\
\hline$G \gamma$ & 2.17500674495 & 4.5 & \\
\hline$W$ & 200.000 & 1416.76626314 & $\mathrm{MeV}$ \\
\hline$c p$ & 644.444581326 & 2160.05810228 & $\mathrm{MeV}$ \\
\hline$E$ & 1.13827204600 & 2.35503830914 & $\mathrm{GeV}$ \\
\hline$B \rho$ & 2.14963573675 & 7.20517826462 & $\mathrm{Tm}$ \\
\hline$\beta$ & 0.566160421483 & 0.917207203761 & \\
\hline$\gamma$ & 1.21315779454 & 2.50997386012 & \\
\hline$\eta$ & -0.6362 & -0.1159 & \\
\hline$h$ & 1 & 1 & \\
\hline$h f$ & 841.166737926 & 1362.77884657 & $\mathrm{kHz}$ \\
\hline$R$ & $201.780 /(2 \pi)$ & $128.4526 / 4$ & $\mathrm{~m}$ \\
\hline
\end{tabular}

Table 2: Polarized Protons in Booster

\begin{tabular}{|c|c|c|c|}
\hline Parameter & $G \gamma=3$ & $G \gamma=4$ & Unit \\
\hline$G \gamma$ & 3.0 & 4.0 & \\
\hline$W$ & 631.753493427 & 1155.09533990 & $\mathrm{MeV}$ \\
\hline$c p$ & 1.25881919359 & 1.87131840691 & $\mathrm{GeV}$ \\
\hline$E$ & 1.57002553943 & 2.09336738590 & $\mathrm{GeV}$ \\
\hline$B \rho$ & 4.19896885328 & 6.24204631230 & $\mathrm{Tm}$ \\
\hline$(B \rho) / \rho$ & 0.302833548731 & 0.450182199999 & $\mathrm{~T}$ \\
\hline$\beta$ & 0.801782621988 & 0.893927372480 & \\
\hline$\gamma$ & 1.67331590675 & 2.23108787566 & \\
\hline$\eta$ & -0.3143 & -0.1581 & \\
\hline$h$ & 1 & 1 & \\
\hline$h f$ & 1.19123988020 & 1.32814294910 & $\mathrm{MHz}$ \\
\hline$R$ & $201.780 /(2 \pi)$ & $201.780 /(2 \pi)$ & $\mathrm{m}$ \\
\hline
\end{tabular}


Table 3: Polarized Protons in AGS for PP-on-Aluminum Stores

\begin{tabular}{|c|c|c|c|c|}
\hline Parameter & Injection & Transition & Extraction & Unit \\
\hline$G \gamma$ & 4.5 & 15.2392025302 & 45.5 & \\
\hline$W$ & 1.41676626314 & 7.03703998500 & 22.8737819686 & $\mathrm{GeV}$ \\
\hline$c p$ & 2.16005810228 & 7.91992760710 & 23.7935613552 & $\mathrm{GeV}$ \\
\hline$E$ & 2.35503830914 & 7.97531239100 & 23.8120540146 & $\mathrm{GeV}$ \\
\hline$B \rho$ & 7.20517826462 & 26.4180348630 & 79.3667776499 & $\mathrm{Tm}$ \\
\hline$\beta$ & 0.917207203761 & 0.993055471537 & 0.999223390833 & \\
\hline$\gamma$ & 2.50997386012 & 8.5000 & 25.3786245857 & \\
\hline$\eta$ & -0.1449 & 0.0 & 0.01229 & \\
\hline$h$ & 12 & 12 & 12 & \\
\hline$h f$ & 4.08833653972 & 4.42642071890 & 4.45370998977 & $\mathrm{MHz}$ \\
\hline$R$ & 128.4526 & 128.4526 & 128.458468103 & $\mathrm{~m}$ \\
\hline
\end{tabular}

Table 4: Polarized Protons in RHIC for PP-on-Aluminum Stores

\begin{tabular}{|c|c|c|c|c|}
\hline Parameter & Injection & $G \gamma=191.5$ & $G \gamma=198.5$ & Unit \\
\hline$G \gamma$ & 45.5 & 191.5 & 198.5 & \\
\hline$W$ & 22.8737819686 & 99.2816915541 & 102.945084479 & $\mathrm{GeV}$ \\
\hline$c p$ & 23.7935613552 & 100.215571393 & 103.879119213 & $\mathrm{GeV}$ \\
\hline$E$ & 23.8120540146 & 100.219963600 & 103.883356525 & $\mathrm{GeV}$ \\
\hline$B \rho$ & 79.3667776499 & 334.283163964 & 346.503444104 & $\mathrm{Tm}$ \\
\hline$\beta$ & 0.999223390833 & 0.999956174327 & 0.999959210867 & \\
\hline$\gamma$ & 25.3786245857 & 106.813332047 & 110.717735830 & \\
\hline$\eta$ & $3.560 \times 10^{-4}$ & $1.8209 \times 10^{-3}$ & $1.8270 \times 10^{-3}$ & \\
\hline$f$ & 78.1352629784 & & 78.1929095053 & $\mathrm{kHz}$ \\
\hline$h$ & 360 & & 360 & \\
\hline$h f$ & 28.1286946722 & & 28.1494474219 & $\mathrm{MHz}$ \\
\hline$\delta C$ & 14.5260 & & 9.2135 & $\mathrm{~mm}$ \\
\hline
\end{tabular}


Table 5: Polarized Protons in RHIC for $250 \mathrm{GeV}$ PP Stores

\begin{tabular}{|c|c|c|c|c|}
\hline Parameter & Injection & $G \gamma=477.5$ & $E=250 \mathrm{GeV}$ & Unit \\
\hline$G \gamma$ & 45.5 & 477.5 & 477.699235564 & \\
\hline$W$ & 22.8737819686 & 248.957459646 & 249.061727954 & $\mathrm{GeV}$ \\
\hline$c p$ & 23.7935613552 & 249.893970242 & 249.998239285 & $\mathrm{GeV}$ \\
\hline$E$ & 23.8120540146 & 249.895731692 & 250 & $\mathrm{GeV}$ \\
\hline$B \rho$ & 79.3667776499 & 833.556560794 & 833.904364882 & $\mathrm{Tm}$ \\
\hline$\beta$ & 0.999223390833 & 0.999992951261 & 0.999992957140 & \\
\hline$\gamma$ & 25.3786245857 & 266.336115157 & 266.447243170 & \\
\hline$\eta$ & $3.560 \times 10^{-4}$ & $1.8945 \times 10^{-3}$ & $1.8945 \times 10^{-3}$ & \\
\hline$h$ & 360 & 360 & 360 & \\
\hline$h f$ & 28.1287707269 & 28.1504646061 & 28.1504647716 & $\mathrm{MHz}$ \\
\hline$\delta C$ & 4.160 & 0.038 & 0.038 & $\mathrm{~mm}$ \\
\hline
\end{tabular}

Table 6: Polarized Protons in RHIC for $255 \mathrm{GeV}$ PP Stores

\begin{tabular}{|c|c|c|c|c|}
\hline Parameter & Injection & $G \gamma=487.0$ & $E=255 \mathrm{GeV}$ & Unit \\
\hline$G \gamma$ & 45.5 & 487.0 & 487.253220275 & \\
\hline$W$ & 22.8737819686 & 253.929207188 & 254.061727954 & $\mathrm{GeV}$ \\
\hline$c p$ & 23.7935613552 & 254.865752145 & 254.998273809 & $\mathrm{GeV}$ \\
\hline$E$ & 23.8120540146 & 254.867479234 & 255 & $\mathrm{GeV}$ \\
\hline$B \rho$ & 79.3667776499 & 850.140640113 & 850.582684802 & $\mathrm{Tm}$ \\
\hline$\beta$ & 0.999223390833 & 0.999993223582 & 0.999993230624 & \\
\hline$\gamma$ & 25.3786245857 & 271.634948862 & 271.776188033 & \\
\hline$\eta$ & $3.560 \times 10^{-4}$ & $1.8950 \times 10^{-3}$ & $1.8950 \times 10^{-3}$ & \\
\hline$h$ & 360 & 360 & 360 & \\
\hline$h f$ & 28.1287707269 & 28.1504722721 & 28.1504724704 & $\mathrm{MHz}$ \\
\hline$\delta C$ & 4.160 & 0.038 & 0.038 & $\mathrm{~mm}$ \\
\hline
\end{tabular}

